\title{
Multiple colonization with highly resistant bacteria: carbapenemase- producing Enterobacteriaceae, carbapenemase-producing Pseudomonas aeruginosa, carbapenemase-producing Acinetobacter baumannii, and glycopeptide-resistant Enterococcus faecium
}

\section{To the Editors,}

The dissemination of carbapenemase-producing bacteria worldwide is an important source of concern because carbapenemase producers are multidrug resistant (Nordmann and Poirel, 2014). National guidelines increasingly recommend a systematic screening of at least carbapenemase-producing Enterobacteriaceae (CPE) and glycopeptideresistant enterococci (GRE) in patients admitted to hospitals who have been hospitalized aboard during the preceding 12 months (Lepelletier et al., 2011). We have investigated the occurrence of colonization and infection with multiple highly resistant bacteria of more than 4 different genus in 2 patients directly transferred from a foreign country.

In June 2014, a 33-year-old French man (patient A) was admitted for a suicide attempt in a Vietnamese hospital where he was treated during 10 days for pneumonia with piperacillin + tazobactam before his transfer to Necker-Enfants Malades University Hospital in Paris, France. At the day of his hospitalization in France, distal protected pulmonary samples were collected, and imipenem was administered subsequently to a persistent fever. In addition, systematic screening to detect carbapenemase producers and GRE was also performed. Screening of extended spectrum $\beta$-lactamase (ESBL) producing Enterobacteriaceae, carbapenemase producers, and GRE was done on selective media (bioMérieux, La Balme-les-Grottes, France) ChromID ESBL, ChromID Carba Smart, and VRE medium, respectively. Carbapenemase production was identified using the Carba NP test for Enterobacteriaceae (Dortet et al., 2014a) and Pseudomonas aeruginosa (Dortet et al., 2012) and CarbAcineto NP test for Acinetobacter baumannii (Dortet et al., 2014b). Definitive identifications of resistance determinant were done by PCR amplifications followed by sequencing. Pulmonary samples grew an OXA-23-producing $A$. baumannii isolate and an IMP-1-producing $P$. aeruginosa (Table 1 ). Screening identified also that the patient was colonized with a KPC-2-producing Klebsiella pneumoniae, a CTX-M-15producing K. pneumoniae, and a VanA-positive glycopeptide-resistant Enterococcus faecium (Table 1 ).

Patient B was a 66-year-old French woman who was hospitalized in May 2014 in the intensive care unit of Paris suburb (Saint-Denis, France). She was directly transferred from Morocco, where she was admitted in the intensive care unit consecutively to a road accident. Seven days after her admission, she developed a ventilation-associated pulmonary infection due to a multidrug-resistant $P$. aeruginosa that was treated with imipenem, colistin, metronidazole, and fluconazole for 14 days. Twenty-one days after the admission, the patient developed another pulmonary infection due to a multidrug-resistant $A$. baumannii only susceptible to colistin, resulting in her transfer to the French hospital. At the admission, the systematic screening of CPE using rectal swab samples revealed the presence of an OXA-48-producing $K$. pneumoniae (Table 1). After 24 hours, the patient developed a pyelonephritis due to a VIM-4-producing $P$. aeruginosa and a bronchitis due to an OXA-23-producing A. baumannii (Table 1), which were treated with amikacin, fosfomycin, and colistin. Although apyrexia was

Table 1

Clinical data on the 2 patients hospitalized in France after hospitalization in another country and were carrying multidrug-resistant bacteria.

\begin{tabular}{|c|c|c|c|c|c|}
\hline Patient & Country of initial hospitalization & Species & Clinical sample & $\beta$-Lactamase content ${ }^{\mathrm{a}}$ & Non- $\beta$-lactam resistance determinants \\
\hline \multirow[t]{5}{*}{ A } & Vietnam & A. baumannii & PDP & OXA-23 & ArmA \\
\hline & & P. aeruginosa & PDP & IMP-1 & \\
\hline & & K. pneumoniae & Rectal swab & KPC-2, CTX-M-15, TEM-1, SHV-1, OXA-1 & AAC6'-Ib-cr, QnrB \\
\hline & & K. pneumoniae & Rectal swab & CTX-M-15, TEM-1, SHV-1, OXA-1 & AAC6'-Ib-cr, QnrB \\
\hline & & E. faecium & Rectal swab & None & VanA \\
\hline \multirow[t]{4}{*}{ B } & Morocco & A. baumannii & PDP & OXA-23 & \\
\hline & & P. aeruginosa & Urine and blood culture & VIM-4 & \\
\hline & & P. rettgeri & Urine & NDM-1, CTX-M-15, SHV-12, TEM-1, & AAC6'-Ib-cr, QnrB \\
\hline & & K. pneumoniae & Rectal swab & OXA-48, CTX-M-15, OXA-9 & AAC6'-Ib-cr, QnrB \\
\hline
\end{tabular}

$\mathrm{PDP}=$ distal protected pulmonary sample.

a Carbapenemase are in boldface; ESBL are underlined. 
obtained in 3 days, fever reappeared under colistin treatment. A naturally colistin-resistant Providentia rettgeri isolate producing the NDM-1 carbapenemase was isolated from 3 successive urine samples recovered by urinary catheter (Table 1 ). The urinary catheter was removed, and the patient received 2 doses of amikacin, leading to a rapid apyrexia.

Co-occurrence of multiple resistance determinants in the same species has become a common feature among carbapenemase producers (Compain et al., 2014). Nevertheless, co-occurrence in the same patient of a least 4 different highly resistant bacterial species is worrisome because it might potentially lead to a real therapeutic deadlock.

\section{Acknowledgments}

This work was partially funded by a grant from the INSERM (U914).

Delphine Girlich Faculty of Medecine, South-Paris University, Le Kremlin-Bicêtre, France

Patrice Nordmann INSERM U914 " Emerging Resistance to Antibiotic » Le Kremlin-Bicêtre, France Associated National Reference Center to Antibiotic Resistance, Le Kremlin-Bicêtre, France Medical and Microbiology Unit, Department of Medecine University of Fribourg, Fribourg, Switzerland Hôpital Fribourgeois-hôpital Cantonal, Fribourg, Switzerland

Hervé Lécuyer Patrick Berche

Bacteriology-Virology-Hygiene unit, Necker-Enfants Malades Hospital Assistance Publique / Hôpitaux de Paris, Paris, France

\author{
Anne Marmorat-Khuong \\ Isabelle Gros \\ Centre Hospitalier de Saint-Denis \\ Saint-Denis, France
}

Gaëlle Cuzon

Bacteriology-Hygiene unit, Bicêtre Hospital Assistance Publique / Hôpitaux de Paris, Le 11 Kremlin-Bicêtre, France INSERM U914 " Emerging Resistance to Antibiotic " Le Kremlin-Bicêtre, France Associated National Reference Center to Antibiotic Resistance, Le Kremlin-Bicêtre, France

Laurent Dortet Faculty of Medecine, South-Paris University, Le Kremlin-Bicêtre, France Bacteriology-Hygiene unit, Bicêtre Hospital Assistance Publique / Hôpitaux de Paris, Le Kremlin-Bicêtre, France INSERM U914 " Emerging Resistance to Antibiotic "

Le Kremlin-Bicêtre, France

Associated National Reference Center to Antibiotic Resistance, Le Kremlin-Bicêtre, France Corresponding author. Mailing address: Service de BactériologieVirologie, Hôpital de Bicêtre, 78 rue du Général Leclerc, 94275 Le Kremlin-Bicêtre Cedex, France E-mail address: laurent.dortet@bct.aphp.fr

\section{References}

Compain F, Decre D, Frazier I, Ramahefasolo A, Lavollay M, Carbonnelle E, et al Carbapenemase-producing bacteria in patients hospitalized abroad, France. Emerg Infect Dis 2014;20:1246-8.

Dortet L, Poirel L, Nordmann P. Rapid detection of carbapenemase-producing Pseudomonas spp. J Clin Microbiol 2012;50:3773-6.

Dortet L, Brechard L, Poirel L, Nordmann P. Impact of the isolation medium for detection of carbapenemase-producing Enterobacteriaceae using an updated version of the Carba NP test. J Med Microbiol 2014a;63:772-6.

Dortet L, Poirel L, Errera C, Nordmann P. CarbAcineto NP test for rapid detection of carbapenemase-producing Acinetobacter spp. J Clin Microbiol 2014b;52:2359-64.

Lepelletier D, Andremont A, Grandbastien B, National Working G.. Risk of highly resistant bacteria importation from repatriates and travelers hospitalized in foreign countries: about the French recommendations to limit their spread. JTravel Med 2011;18:344-51.

Nordmann P, Poirel L. The difficult-to-control spread of carbapenemase producers among Enterobacteriaceae worldwide. Clin Microbiol Infect 2014;20:821-30. 